\title{
Multiple dural arteriovenous fistulae and SARS CoV-2, a lethal combination
}

\author{
Carlos G. Seañez*, Jesus A. Morales, Valeria González, Luis F. Checca, and \\ Angel R. Martínez-Ponce de León \\ Neurosurgery and Neurologic Endovascular Therapy Service, "Dr. Jose E. Gonzalez" University Hospital, Universidad Autonóma de Nuevo León, \\ Monterrey, Nuevo Leon, Mexico
}

\begin{abstract}
Introduction: Dural arteriovenous fistulas are abnormal communications between the arterial and dural sinus vessels or the cortical veins. Multiple dural arteriovenous fistulae have an incidence rate of 6-9\%. Their pathophysiology is poorly understood, although there is a consensus on three theories for their formation. The systemic infection caused by SARS-CoV-2 in its severe presentations is associated with a pro-inflammatory state that represents an ideal condition for complications of pre-existing cerebrovascular diseases. Digital subtraction angiography is considered the gold standard to understand the architecture of this lesion. Case Presentation: The patient was a 49-year-old male with a history of multiple head trauma, a 20-year history of tobacco use, tinnitus, and gradual deterioration of higher mental functions. On admission, he presented respiratory distress and was dependent on oxygen. Conclusion: This case presents a bleak picture of the overlap between two devastating diseases as a clear example of the direct and collateral damage of the SARS-CoV-2 pandemic. Coronavirus disease in 2019 does not exclude a previous underlying pathology.
\end{abstract}

Key words: Multiple dural arteriovenous fistulae. SARS-CoV-2. Dural venous sinuses. Cortical veins.

\section{Introduction}

Dural arteriovenous fistulae (DAVFs) are abnormal communications between the meningeal arterial vessels and the dural sinuses or cerebral cortical veins. At the start of their study, they were categorized as a variant of arteriovenous malformations, but unlike these, they represent a non-congenital-acquired pathology1.

Multiple DAVF (MDAVF) have an incidence rate of $6-9 \%$ of all DAVF ${ }^{2,3}$. Their pathophysiological mechanism is poorly understood. Associated risk factors such as hypercoagulable states have been included. There is a consensus that three mechanisms exist for their formation: (1) they may develop after partial or total recanalization of multiple dural sinus thrombosis; (2) DAVF can induce dural sinus thrombosis or venous hypertension, resulting in the formation of MDAVFs; and (3) MDAVF can be the product of angiogenic activity associated with an underlying or concealed disease.

Systemic SARS-CoV-2 infection in its severe presentations is associated with a pro-inflammatory state with impaired coagulation. This pro-inflammatory state is an ideal setting for the complication of pre-existing cerebrovascular conditions. It has been previously described how neurological manifestations may represent the predominant clinical picture in SARS-CoV-2-positive patients, especially severe coronavirus disease in 2019 (COVID-19) ${ }^{4,5}$.

\section{Correspondence:}

${ }^{*}$ Carlos G. Seañez

E-mail: carlosseanez@ hotmail.com
Available online: $01-12-2021$

Date of reception: 30-03-2021

Date of acceptance: 20-07-2021 DOI: 10.24875/RMU.21000022
Medicina Universitaria. 2021;23(4):148-152 www.medicinauniversitaria.org

作 license (http://creativecommons.org/licenses/by-nc-nd/4.0/). 
According to the nomenclature used by Guo et al. ${ }^{6}$ for classification, when multiple fistulae are present simultaneously, they are called "synchronous." When a patient presents a dural fistula in a certain location and subsequently develops multiple fistulae, they are called "metachronous," which are more frequent than synchronous ones.

Regarding the clinical characteristics, the symptoms can be mild, or "benign," or rapidly progressive; the latter are classified as "malignant." These symptoms include rapidly progressive neurological deterioration, focal neurological deficits, seizures, and intracranial hemorrhage, which are related to intracranial venous hypertension due to "arterialization" and retrograde flow in the intracranial cortical veins.

Digital subtraction angiography is considered the gold standard to understand the architecture of the lesion, the feeding pedicles to the fistula, the venous pattern, and the location and to observe the speed and direction of the flows. These characteristics are not obtained through computed tomography and magnetic resonance, even with current techniques.

In patients with atypical pneumonia with neurological manifestations, the first clinical suspicion should be cerebrovascular disease. We present a patient with the association of pre-existing neurovascular pathology and the presence of atypical pneumonia due to SARS-CoV-2.

\section{Case presentation}

The patient was a 49-year-old male, a furniture store merchandise delivery man with a history of multiple head trauma. The last head trauma was more than 7 years ago after a fall from a ladder approximately $2.5 \mathrm{~m}$ high with a head impact and a loss of alertness without requiring surgical treatment. A family member mentioned tobacco use of 20 years, pulsatile tinnitus with at least 5 years of evolution, and a gradual deterioration of higher mental functions during the past 15 days before admission.

The patient presented headache, nausea, vomiting, and altered alertness accompanied by respiratory distress. He was transferred by a relative to the emergency service of a local hospital, being assessed and referred to our unit due to the COVID-19 contingency and after clinical suspicion of pneumonia.

Physical examination on admission revealed a palpable thrill and a holocranial murmur, conjunctival injection, and oxygen-dependent respiratory distress treated by mask at $7 \mathrm{~L} / \mathrm{min}$. On neurological examination, the patient presented a 13-point Glasgow scale coma with disorientation and drowsiness, altered higher mental functions with an inability to perform simple calculations, and impaired short-term memory, $3 \mathrm{~mm}$ isochoric pupils, and papilledema on fundoscopy. The rest of the cranial nerves did not show abnormalities; his muscle strength was preserved at $+4 / 5$ in the four extremities, and his tendon reflexes were increased with no pathological reflexes.

According to the clinical findings, a PCR was requested because of the clinical suspicion of COVID-19; the study was positive, and chest and cranial imaging studies were performed (Figs. 1-3).

During his hospitalization, he presented progressive clinical deterioration due to lung and heart failure. He was admitted to the specialized unit for COVID-19, where he required orotracheal intubation and vasopressors to maintain his blood pressure. The patient continued with hemodynamic instability, which prevented a therapeutic approach to resolve synchronous multiple dural fistulae. He subsequently passed away.

\section{Discussion}

Since the appearance of the first case of the COVID-19, a pandemic has emerged, affecting millions of people worldwide. We present a patient with previous cerebrovascular disease and a SARS-CoV-2 infection. The clinical course of COVID-19 ranges from asymptomatic infection to severe acute respiratory distress with multiorgan involvement and death. At present, there is convincing evidence that SARS-CoV-2 can involve the nervous system, and its neurotropic potential is becoming increasingly well established. In addition, patients with MDAVF have a mean age of 45 years, compared with 53 years for patients with a single dural fistula ${ }^{1}$. Furthermore, patients with rapidly progressive clinical characteristics tend to be younger, which coincides with our patient's age and type of presentation.

Regarding angioarchitecture, dilation of the dural venous sinuses and the cortical veins is almost always linked to rapid progression. Synchronous multiple fistulae are associated with aggressive clinical progres$\operatorname{sion}^{1,7,8}$. In our case, the association of pre-existing neurovascular pathology with severe clinical and radiological characteristics and the presence of atypical pneumonia due to SARS-CoV-2 led to a fatal outcome before we could provide treatment to resolve the fistula.

As previously reported ${ }^{4,5,9}$, in patients with SARSCoV-2 infections with neurological manifestations, the 


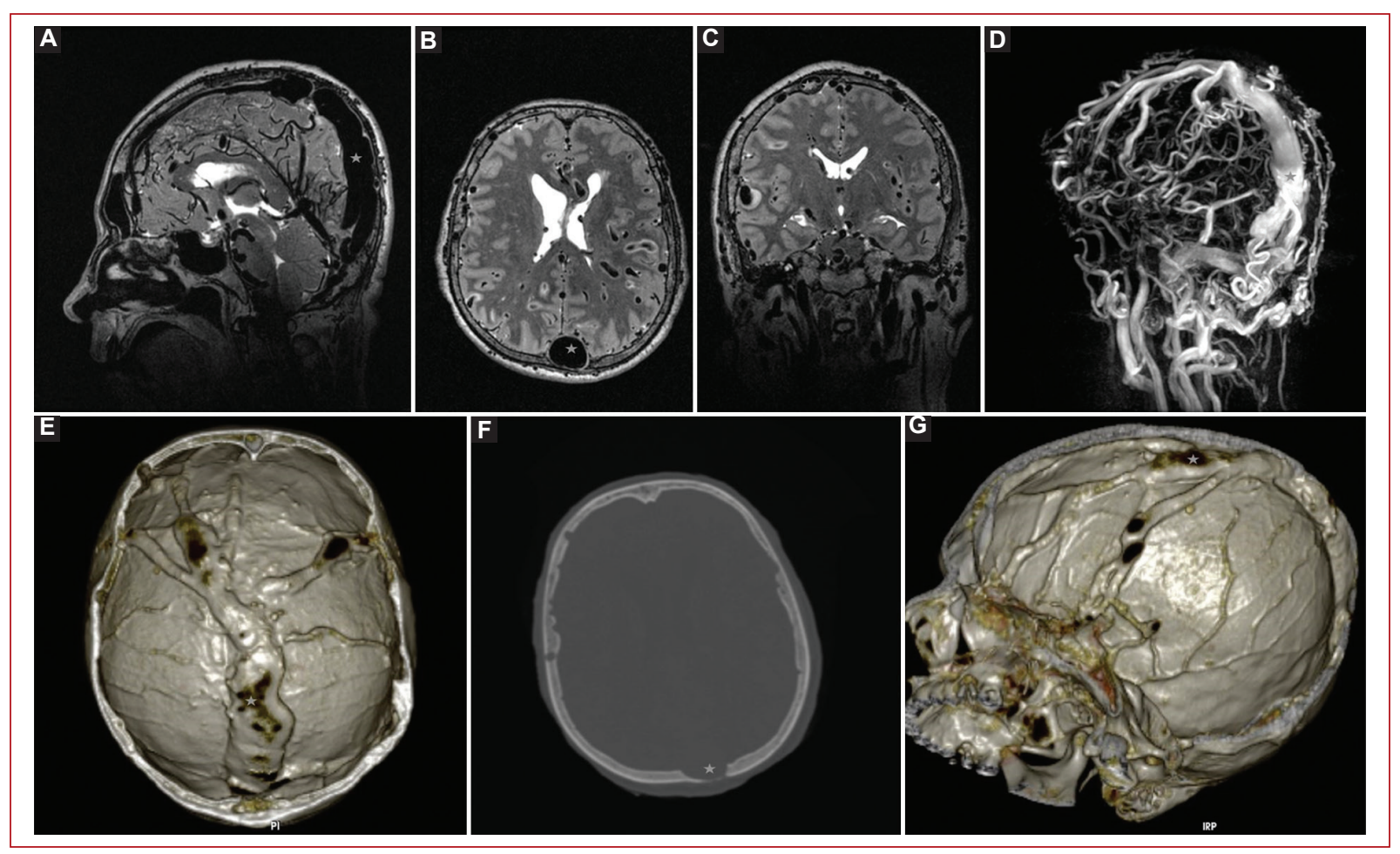

Figure 1. Magnetic resonance imaging (MRI) and cranial tomography (CT). A: MRI FRFSE sequence: Sagittal view shows dilation of the superior longitudinal sinus (SLS), multiple tortuous hypointense images corresponding to arterialized cortical veins (ACV), widened veins adjacent to the brainstem. B: MRI FRFSE sequence: Axial view, multiple tortuous images corresponding to ACV, SLS with increased dimensions, an asymmetric ventricular system as indirect evidence of chronic intracranial hypertension. C: MRI FRFSE sequence: Coronal view, SLS is striking with data of thrombosis and partial recanalization, as well as widened cortical veins. D: Reconstruction of MRI angiography observing multiple extra- and intra-cranial vessels with the direct flow toward dural sinuses, predominantly the SLS. E: Lower to upper view of 3D bone CT reconstruction, where multiple intracranial grooves and indentations are observed due to high-pressure arterialized venous flow. F: Bone CT axial view showing intracranial grooves and indentations as a result of high-pressure arterialized venous flow. G: Side view of 3D bone CT reconstruction with midline section, where multiple intracranial grooves and indentations are observed due to arterialized venous flow of high pressure.

first clinical suspicion should be to rule out cerebrovascular disease since it is associated to a greater extent with prothrombotic effects and, on other occasions, multifocal hemorrhage. In our patient, the neurological deterioration was due to the exacerbation of a pathology with high morbidity and severe radiological characteristics.

Increased blood pressure, factors that affect clotting pathways, and the so-called "cytokine storm" syndrome may increase risk and cerebrovascular complications with high mortality ${ }^{10}$.

Acute respiratory infections of viral and bacterial etiologies are well-recognized triggers of cardiovascular events. Another mechanism that potentially mediates cardiovascular disease from COVID-19 involves the angiotensin-converting enzyme inhibitors pathway ${ }^{11}$.
Pranata et al. found that cerebrovascular and cardiovascular diseases were associated with an increased poor outcome in COVID-19. The association was not influenced by gender, age, hypertension, diabetes, or respiratory comorbidities ${ }^{11}$. A study by Hernández-Fernández et al. included five cases of subarachnoid hemorrhage and single or multiple intraparenchymal hematomas ${ }^{12}$. A single-center observation showed that in 221 COVID-19 patients, 5\% developed acute ischemic stroke, $0.5 \%$ developed cerebral venous sinus thrombosis, and $0.5 \%$ developed cerebral hemorrhage $^{13}$. Dogra et al. found that 28-day mortality was $100 \%$ in large parenchymal hemorrhages with COVID-19, which corresponds to the fatal outcome of our patient ${ }^{14}$. As already described, COVID-19 infection may, in some cases, have acted as a contributing cause 


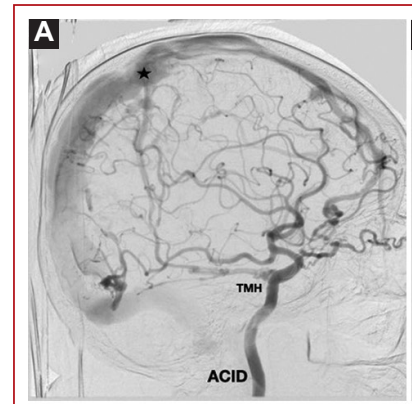

E
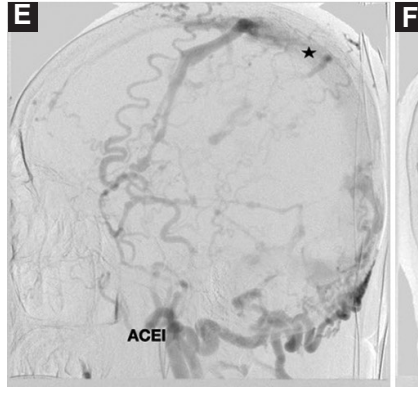
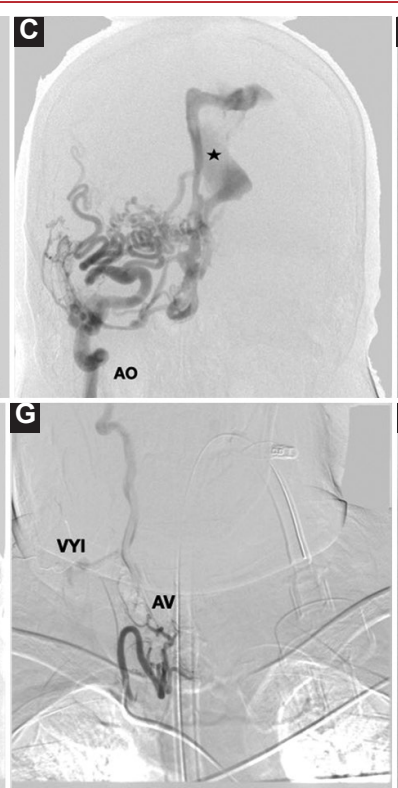
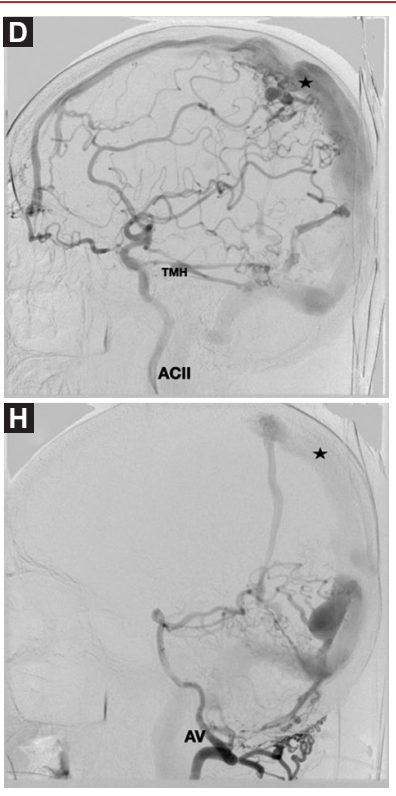

Figure 2. Digital subtraction angiography. A: RICA, lateral view; a thickened superior longitudinal sinus is seen in early arterial phase; a thickened MHT is seen with a fistula of tentorial branches toward the distal third of the SLS and the junction between the transverse and sigmoid sinuses; a dural fistula of the anterior ethmoid branches dependent on the ophthalmic artery toward the proximal third of the SLS is observed B: LICA, anteroposterior view; a large SLS is observed with the destruction of the internal table of the skull. C: Right occipital artery; multiple tortuous distal branches are observed with tributaries toward the SLS in its distal third. D: LICA lateral view; mirror findings of the contralateral artery with the anterior and posterior fistulous branches. E: LECA, a generalized increase in terminal branches with the SLS and the transverse sinus are visible in the early phase. F: Right OA, lateral view. G: Right thyrocervical trunk, viewed from the subclavian artery; an arteriovenous fistula is observed toward the internal jugular vein with a high flow path. H: Left vertebral artery lateral view; a dural fistula is observed with a turn toward the torcula, multiple small branches toward the transverse sinus, and a thickened direct tributary toward the SLS by distal branches of the superior cerebellar and posterior cerebral arteries.

NSLS. MHT: meningohypophyseal trunk; RICA: right internal carotid artery; LICA: left internal carotid artery; OA: occipital artery, LECA: left external carotid artery; IJV: internal jugular vein; VA: vertebral artery.

trSLS.

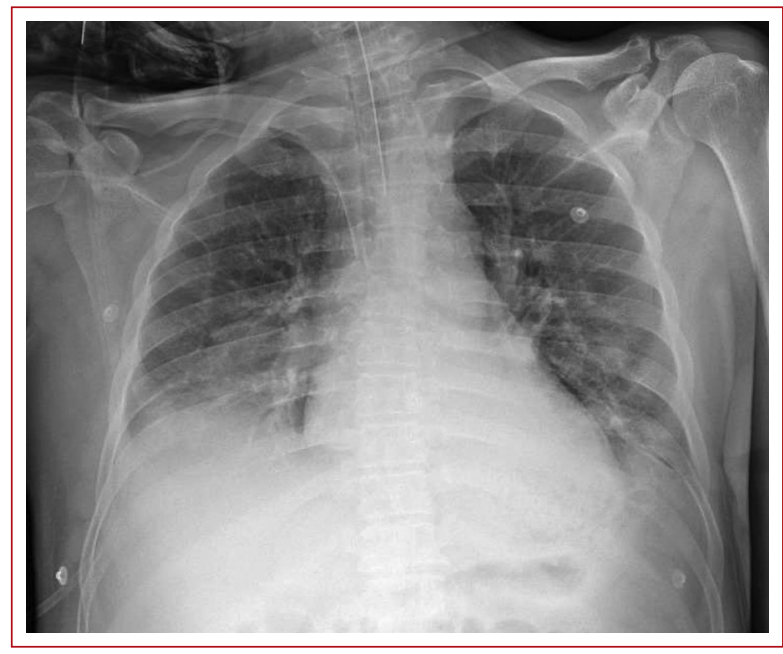

Figure 3. Chest X-ray, with bilateral peripheral, infiltrates. or trigger for the worsening of previously established neurovascular diseases ${ }^{15,16}$.

\section{Conclusion}

This case presents the bleak outlook of two simultaneous devastating diseases as a clear example of the direct and indirect damage; the SARS-CoV-2 pandemic is causing globally. Management requires multidisciplinary participation and a high clinical suspicion for a proper approach to our patients. We must remember that the diagnosis of COVID-19 does not exclude a previous underlying pathology.

\section{Funding}

None. 


\section{Conflicts of interest}

The authors declare that they have no conflicts of interest.

\section{Ethical disclosures}

Protection of human and animal subjects. The authors declare that no experiments were performed on humans or animals for this study.

Confidentiality of data. The authors declare that no patient data appears in this article.

Right to privacy and informed consent. The authors declare that no patient data appears in this article.

\section{References}

1. Hetts SW, Tsai T, Cooke DL, Amans MR, Settecase F, Moftakhar P, et al. Progressive versus nonprogressive intracranial dural arteriovenous fistulae: characteristics and outcomes. AJNR Am J Neuroradiol. 2015;36:1912-9.

2. Barnwell SL, Halbach VV, Dowd CF, Higashida RT, Hieshima GB, Wilson CB. Multiple dural arteriovenous fistulae of the cranium and spine. AJNR Am J Neuroradiol. 1991:12:441-5.

3. Ha SY, Kwon YS, Kim BM, Kim DI, Kim DJ. Clinical and angiographic characteristics of multiple dural arteriovenous shunts. AJNR Am J Neuroradiol. 2012;33:1691-5.

4. Ballvé-Martín A, Boned S, Rubiera M. Complicación trombótica de neumonía grave por COVID-19: ictus por embolismo paradójico atípico [Thrombotic complication of severe COVID-19 pneumonia: stroke due to atypical paradoxical embolism]. Rev Neurol. 2020;71:186-90.
5. Dahl-Cruz F, Guevara-Dalrymple N, López-Hernández N. Trombosis venosa cerebral e infección por SARS-CoV-2 [Cerebral venous thrombosis and SARS-CoV-2 infection]. Rev Neurol. 2020;70:391-2.

6. Guo Y, Yu J, Zhao Y, Yu J. Progress in research on intracranial multiple dural arteriovenous fistulas. Biomed Rep. 2018;8:17-25.

7. van Dijk JM, terBrugge KG, Willinsky RA, Wallace MC. Clinical course of cranial dural arteriovenous fistulas with long-term persistent cortical venous reflux. Stroke. 2002;33:1233-6.

8. Hu YC, Newman CB, Dashti SR, Albuquerque FC, McDougall CG. Cranial dural arteriovenous fistula: transarterial onyx embolization experience and technical nuances. J Neurointerv Surg 2011;3:5-13.

9. Muro I, Ximénez-Carrillo A, Carreras MT, de la Fuente E, Aizpún A Orts $M$, et al. Hemorragias cerebrales múltiples secundarias a coagulación intravascular diseminada en un paciente con COVID-19 [Multiple cerebral haemorrhages secondary to disseminated intravascular coagulation in a patient with COVID-19]. Rev Neurol. 2021;72:33-4.

10. Carod-Artal FJ. Neurological complications of coronavirus and COVID-19. Rev Neurol. 2020;70:311-22.

11. Pranata R, Huang I, Lim MA, Wahjoepramono EJ, July J. Impact of cerebrovascular and cardiovascular diseases on mortality and severity of COVID-19-systematic review, meta-analysis, and meta-regression. J Stroke Cerebrovasc Dis. 2020;29:104949.

12. Hernández-Fernández F, Valencia HS, Barbella-Aponte RA, Collado-Jiménez R, Ayo-Martín O, Barrena $\mathrm{C}$, et al. Cerebrovascular disease in patients with COVID-19: neuroimaging, histological and clinical description. Brain. 2020;143:3089-103.

13. LiY, Wang M, Zhou Y. Acute cerebrovascular disease following COVID-19: a single center, retrospective, observational study. SSRN Electron J. 2020:5:279-84.

14. Dogra S, Jain R, Cao M, Bilaloglu S, Zagzag D, Hochman S, et al. Hemorrhagic stroke and anticoagulation in COVID-19. J Stroke Cerebrovasc Dis. 2020;29:104984.

15. Fraiman P, Godeiro C Jr., Moro E, Cavallieri F, Zedde M. COVID-19 and cerebrovascular diseases: a systematic review and perspectives for stroke management. Front Neurol. 2020;11:574694.

16. Collantes ME, Espiritu AI, Sy MC, Anlacan VM, Jamora RD. Neurological manifestations in COVID-19 infection: a systematic review and meta-analysis. Can J Neurol Sci. 2021;48:66-76. 